\title{
First aid elements used in potentially toxic $I$ explosive environments in the context of the covid 19 pandemic
}

\author{
Lorand Toth $^{1 *}$, George Artur Găman ${ }^{1}$, Daniel Pupăzan ${ }^{1}$, Cristian Nicolescu ${ }^{1}$ and Cosmin \\ Ilie $^{1}$ \\ ${ }^{1}$ National Institute for Research and Development in Mine Safety and Protection to Explosion - \\ INSEMEX Petroşani, 32-34 G-ral Vasile Milea Street, Petroşani 332047, Romania
}

\begin{abstract}
Accidents are unplanned occurrences that result in injuries, illness, death, and loss of property and/or production. While there is no way to completely eliminate accidents, there are certain plans, preparations, and actions that can be taken to reduce them. First aid is usually a critical part in the management of acute injuries and conditions. The rescuer is the first person with medical training to arrive at the scene of a medical incident and the purpose of his actions is to maintain vital functions, to prevent the occurrence of dangerous complications for the life and health of the victim, before the intervention of medical staff. Despite current concerns about infection with the new coronavirus 2019 (COVID-19), prevention of its spread and treatment, various lesions and conditions unrelated to this virus still occur. Prompt first aid can prevent an additional burden on the health system by taking care of simple ailments at the crime scene, rather than calling the ambulance system or transporting the victim to a hospital. This paper presents first aid elements in the context of the COVID-19 pandemic, highlighting the manoeuvres that will be performed minimizing the risk of infection with the SARS-CoV-2 pathogen.
\end{abstract}

\section{Introduction}

Every year, around 5 million people in the European Union suffer accidents at work with consequences that are sometimes permanent, and affect people's ability to work and have a life outside work.

Work-related accidents occur in all sectors and include slips, strikes, falls, falls from heights, sharp cuts, burns, electric shocks, traffic accidents on public roads, acute intoxications, contamination, contact with carcinogens, etc. However, many of these problems can be prevented through proper management [1].

Given that accidents at work are the consequence of malfunctions in the work ecosystem and that these dysfunctions can occur at different levels of the work system (structure, functions), measures to prevent accidents at work involve, on one hand, identification of risk

\footnotetext{
* Corresponding author: lorand.toth@insemex.ro
} 
factors for accidents and, on the other hand, measures to remove risk factors or to annihilate their action.

Starting from the four components that structure the work system, measures for prevention of work accidents address the risk factors specific to workers, the risk factors in the work environment as well as the risk factors specific to workload.

\subsection{Organizational and security principles for interventions in hazardous environments}

Ensuring the success of interventions in emergency cases requires solving, first of all, the issue imposed by the presence of hazardous environments. The evolutionary nature of emergencies doesn't even require a prior check of hazards specific to the environments they generated, which, however, are constantly changing and must be considered as if they were always in the specific area of danger [2].

The issues raised by intervention, in order to control an emergency, do not allow a momentary analysis, "in effect", of dangers posed by the environments in which it is necessary to intervene, therefore they must be approached as if they were permanently at a maximum level of danger. Such an approach has the advantage of ensuring a large degree of protection, even for unforeseen situations of sudden increases in concentration limits of hazardous environments in early stages of formation. However, this approach must be based on a very thorough prior analysis of technological processes and all adjacent areas where damage may potentially extend, in terms of hazardous substances that may underlie the occurrence of hazardous environments during the accident. In other words, in addressing hazardous environments generated by emergencies, in order to organize interventions, the "qualitative character" prevails, respectively nature of structural components, to detriment of the "quantitative character", respectively concentration range of components.

In general, an emergency is dangerous because of the effects through which it interacts with the environment. Conclusions regarding the effects of emergencies and the way in which they affect, through consequences, the full potential danger, show that relative to intervention actions, it is increased. Most of the increasing is caused by rising the value of existing potential partial hazards generated by hazardous environments, by the fact that intervention forces act in direct contact with them, and by the emergence of new potential partial hazards relative to intervention personnel, as a result of electrical safety and those related to bodily integrity (dangers of injury, fall, crushing, mechanical shocks, etc.).

In order to be able to solve the overall problems of interventions, a systemic analysis of the specificity of each intervention, classified according to nature of the hazardous environment of intervention, is necessary. This means analysing the manner in which potential danger manifests in the case of interventions in explosive, toxic, complex and aggressive environments (with chemical and thermal variants), and finding solutions to mitigate them.

\subsection{Intervention in explosive environments}

Intervention in explosive environments is a necessity that can easily arise in case of breakdowns in industrial installations producing or using specific chemicals or in the supply of specific fuels in other industrial aggregates. The presence of explosive environments in certain areas of these installations is a matter of normality and is technologically acceptable under normal operating conditions, further prove that, damage in these areas will increase the occurrence and amplitude of these hazardous environments [3]. Acceptance of the presence of explosive environments, under the conditions of a normal technological process, involves a set of framework measures to prevent the occurrence of triggers for this type of 
environment. This is ensured by designing and maintaining electrical installations and equipment in adequate protection, so as not to generate electrical sparks with sufficient energy to initiate the explosion, as well as by grounding all elements of the installation that could accumulate electrostatic energy, while providing antistatic protective equipment [4].

As a result of measures presented above, in case of interventions for eliminating damages in explosive environments, the problem of avoiding the occurrence of mechanical sparks remains to be solved [5].

It is obvious that mechanical sparks should be avoided during interventions in explosive environments. This is achievable by:

- special attention when handling metal joinery;

- exclusive use of special tools, made of metals or alloys that do not produce sparks able of igniting explosive mixtures (bronze, copper, beryllium);

- presumably areas of impact or friction should be protected with materials that do not produce sparks;

- avoiding the impact produced between steel and aluminium, by interposing another material on the exposed areas.

In addition to these measures, care must be taken not to bring and use portable electrically operated devices and tools that are not constructively suitable for use in explosive environments, during such interventions.

\subsection{Intervention in toxic environments}

Toxic environment is relatively easy to find in industrial installations (steel, chemistry, petrochemistry, etc.). One of the chemical compounds that occurs most easily, through any incomplete combustion process, is carbon monoxide, an extremely toxic gas, but also other substances present or resulting from industrial technologies, which, if inhaled or swallowed, have toxic characteristics [6].

Most of chemically aggressive substances that cause, by inhalation, serious damage to bronchi, leading to pulmonary oedema, also have toxic effects, as a result of ingestion.

A very important element for intervention action is the need to consider as toxic environments that cause asphyxia, either because of the lack of minimum required oxygen concentration $(17 \% \mathrm{vol})$, or because of the presence of high concentration of carbon dioxide, substance harmless in itself, but which can temporarily cause dizziness and loss of consciousness without having intoxicating effects. Usually, these two situations are associated in the case of fires in confined spaces, when oxygen concentration decreases greatly, instead releasing carbon dioxide in appreciable quantities, as a product of complete combustion, accompanied also by other combustion products (carbon monoxide, smoke, etc.).

The toxic environment can only be the result of a wrong technological manoeuvre or a damage, the effect of which was the release into the atmosphere of a quantity of toxic substance that resulted in concentrations higher than the maximum allowed ones, in the area. [7]. A particular case of toxic environment is fires. Violent burns caused by fires, in addition to massive release of combustion products (mostly toxic), are worryingly reducing the percentage of oxygen in burned and surrounding areas.

Interventions in toxic environments must solve, according to emergency technological instructions, the issues of restoring installations that release toxic substances to normal parameters and, if toxic substances are the result of fires, the interventions must deal with their extinction.

Interventions must be carried out in complete safety for the intervention personnel, by using effective respiratory protection equipment (insulating devices), as well as other assortments of protective equipment, appropriate to other possible hazardous characteristics 
manifested by toxic environments (chemical and thermal aggression, possibly explosiveness).

Intervention in toxic environments can very often take the form of rescuing people caught in toxic environments, requiring immediate first aid.

\subsection{Intervention in complex hazardous environments}

Situations presented above are rare in practice. When a breakdown occurs in an industrial technological installation, it simultaneously generates several types of hazardous environments, often even all the types studied, namely those covered by fig. 1 [8].

\begin{tabular}{|c|c|c|c|c|c|c|}
\hline \multicolumn{7}{|c|}{$\begin{array}{l}\text { DANGEROUS ENVIRONMENTS } \\
\text { consisting of: }\end{array}$} \\
\hline \multicolumn{3}{|c|}{\begin{tabular}{l|l} 
ONE DANGEROUS SUBSTANCE \\
\end{tabular}} & \multicolumn{4}{|c|}{ MORE DANGEROUS SUBSTANCES } \\
\hline \multicolumn{2}{|c|}{$\begin{array}{l}\text { SINGLE DANGER } \\
\text { CHARACTERISTICS }\end{array}$} & $\begin{array}{l}\text { MORE DIFFERENT } \\
\text { DANGER } \\
\text { CHARACTERISTICS }\end{array}$ & \multicolumn{3}{|c|}{$\begin{array}{c}\text { SINGLE COMMON } \\
\text { DANGEROUS } \\
\text { CHARACTERISTICS }\end{array}$} & $\begin{array}{c}\text { ONE OR MORE } \\
\text { DIFFERENT } \\
\text { DANGER } \\
\text { CHARACTERISTIC } \\
\mathrm{S}\end{array}$ \\
\hline \multicolumn{2}{|c|}{$\begin{array}{c}\text { SIMPLE } \\
\text { ELEMENTAL } \\
\text { ENVIRONMENTS }\end{array}$} & $\begin{array}{c}\text { COMPLEX } \\
\text { ELEMENTAL } \\
\text { ENVIRONMENTS }\end{array}$ & \multicolumn{3}{|c|}{$\begin{array}{c}\text { SIMPLE } \\
\text { COMPOUND } \\
\text { ENVIRONMENTS }\end{array}$} & $\begin{array}{c}\text { COMPLEX } \\
\text { COMPOUND } \\
\text { ENVIRONMENTS }\end{array}$ \\
\hline 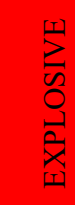 & 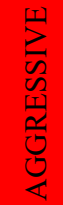 & & $\begin{array}{l}\sum_{\infty}^{1} \\
0 \\
0 \\
\stackrel{1}{\mathbf{a}} \\
\text { II }\end{array}$ & 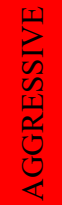 & 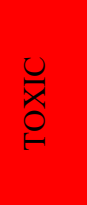 & \\
\hline THER & $\mathrm{C}$ Cl & & THER & $\mathrm{IC} \mathrm{C}$ & MICAI & \\
\hline
\end{tabular}

Fig. 1. Classification of hazardous environments according to hazardous characteristics of substances in their composition.

Depending on the type of environment resulting in such situations, respectively complex hazardous environment, interventions in such cases are called interventions in complex hazardous environments. This is because, in addition to the complexity of the environment generated, in most cases a significant deterioration of visibility is added in the affected areas, scenes of intervention.

Experience taught us that most complex situations are those that arise during emergencies and, simultaneously with their evolution, trigger and develop fires/explosions. These situations are characterized by the presence, in addition to the toxic environment, of an environment with significant thermal aggression, along with massive smoke emissions that reduce visibility to zero.

According to the above, fire intervention is an intervention that takes place in particularly complex situations, situations that can be described by the following main characteristics:

- presence of toxic environments (quite often also chemically aggressive);

- presence of flames and, generally, of a thermally aggressive environment;

- presence of dense smoke that minimizes visibility;

- presence of incandescent liquids, flowing in undetermined directions;

- presence of people trapped in rooms exposed to dangers, located in hard-to-reach places; 
-presence, in immediate vicinity, of combustible or explosive substances that may favour triggering of explosions or spread of fires.

Intervention, under such conditions, presumes, first of all, an excellent physical as well as theoretical and practical training of the specialized intervention personnel, training acquired through the most complex practical exercises, with simulation of possible emergencies [9]. Making an inventory of preliminary measures necessary for the special training carried out by specialized intervention teams, in order to solve the issues of interventions in complex situations, the following results:

- recognition of objectives and installations in which technological breakdowns create complex situations, up to accustoming of intervention personnel with them and their surroundings;

- creating tactical situations as diverse and complex as possible, establishing characteristic intervention methods and training personnel until the intervention steps become a reflex;

- appropriate endowment of intervention personnel with adequate protective equipment and building, through trainings, the reflex skills necessary for its efficient use;

- building, through training, the reflex skills for quick handling of all technical means useful for mitigating damage and its consequences;

- building, through training, the physical condition and endurance needed to cope with special efforts in hostile environmental conditions.

\section{Organizational and safety principles of emergency interventions}

Approach of an intervention, from a conceptual point of view, respectively drafting the intervention plan, in addition to its development framework and its problematic framework (emergency's specificity), must come under a main set that meets fundamental requirements common to all interventions, requirements that materialize in principles [10].

Considering life safety as the "zero" priority for any intervention, followed by that of security of infrastructure and technological installations, this paper also addresses a set of security principles.

Organizational principles of emergency interventions:

- Need for anticipation;

- Planning the intervention must take into account the fact that it is, structurally, a cooperative action;

- Adaptability of present objective conditions;

- The possibility of redirecting forces with minimum effort and maximum operability;

- Full use of local resources and effective engagement of as few as possible forces, with priority respect for the intervention's security principles;

- Restoring the technological installations to their initial functional parameters.

In drawing up intervention plans for technological breakdowns, it must be borne in mind that intervention actions, taking place in hazardous environments, induce a high degree of insecurity for intervention teams, which must be counterbalanced by the implementation of security principles [11].

Protecting and saving human lives underpins the following security principles of interventions:

- Protecting and saving lives is the "zero" priority goal of intervention actions;

- Safety of intervention forces within the activities carried out, is a condition for effectiveness of interventions;

- Safety of buildings and installations threatened by the effects of damage is a condition for persistency and security of intervention. 


\subsection{Set-up and basic principles in providing first aid}

Emergencies are materialized by states of serious alteration of body's health, occurred as a result of the brutal action of a harmful factor on bodily integrity [5].

First aid is a complex of emergency measures, which is applied in cases of accidents, intoxications or illnesses, before the intervention of specialized medical staff.

Setting up first aid at the scene of the accident is of particular importance for rescuing the injured [12].

1. First of all, the necessary measures shall be taken to create a disciplined climate, by controlling panic and congestion around the injured party. Simultaneously with the provision of first aid, measures will be taken to notify the emergency service by calling the unique number "112".

2. Setting lose the injured person. The injured person will be spared as much as possible. Brutal gestures and excessive mobilization will be avoided. The removal of the injured person from the rubble or from the damaged spaces will be performed with the greatest gentleness, proceeding by methodical release, if necessary, with the sacrifice of the materials covering $\operatorname{him} /$ her.

It is forbidden to pull on the visible parts (limbs, head, clothes, etc.) to set lose the injured person. First of all, the head, face and chest will be released, in order to create the possibility to institute artificial respiration and external cardiac massage, if necessary. The spine and head should be left, as much as possible, in the position they were initially found in. During immobilization of the victim, the head-neck-chest axis will be kept stabile.

Excessive mobilization of injured persons is the main cause of shock and complications.

3. After release, the victim will be gently laid on a blanket / stretcher. It will be placed in supine position, with the head placed at the same level as the chest.

4. Seriously injured people are usually unconscious. The first gesture rescuers will be to control breathing and heartbeat. Breath testing, in pandemic context, will be performed by watching the victim's chest, to identify normal breathing. To minimize the risk of infection, the airways will not be opened and we will not approach the victim's face, mouth / nose.

The main duty of any rescuer is to help or maintain the major functions of the human body [13].

Injured persons with apparent signs of death will not be abandoned, as they can be saved by rapidly performing artificial respiration and external cardiac massage. Until the arrival of the ambulance or medical staff, seriously injured persons will not be moved or displaced unnecessarily, but only as much as necessary to establish measures to maintain vital functions (breathing and blood circulation).

5. Depending on the condition of the injured person, first aid measures shall be instituted in a certain order:

- External cardiac massage;

- Artificial respiration (with the help of a balloon or a pulmonary motor);

Stopped breathing is followed, in a few minutes, by heart stop. Also, cardiac arrest is quickly followed by stopped breathing. The injured person's life is decided at this moment, because the unrectified cardio-respiratory arrest causes, after 3 minutes from installation, serious injuries in vital organs, incompatible with life.

- Stopping bleeding;

- Wound dressing;

- Immobilization of fractures.

In most cases, first aid decides the success of emergency care and the recovery of injured.

6. Ensuring fast and non-traumatic transport.

If it is necessary to provide first aid at the same time to a large number of injured persons, a triage of cases must be carried out, depending on the condition of each, as follows: 
- first emergency cases are those in which the injured person presents cardio-respiratory arrest, large haemorrhages, which cannot be stopped by tourniquet, haemorrhages of the internal organs, large wounds in the lungs, state of shock;

- cases of second emergency, are represented by injuries with arterial haemorrhages that can be stopped by tourniquet, large abdominal wounds, amputations of limbs and large bone and muscle damage, injured that have lost consciousness;

-third degree emergencies are represented by injuries with craniocerebral, vertebramedullary and pelvic traumas, accompanied by fractures and injuries of the internal organs, open fractures, deep wounds, haemorrhages;

- the other injured fall into the category of ordinary emergencies. Depending on the category of emergency, first aid is provided and transport of injured is ensured.

\subsection{First aid during the COVID-19 pandemic}

The World Health Organization has declared COVID-19 a pandemic. It brings a global crisis that causes significant mortality and morbidity rates in many regions. The new Corona virus 2 (SARS-CoV-2), the etiological agent, is highly contagious and, in the absence of population immunity, has a much higher mortality compared to seasonal influenza, especially for the vulnerable.

Despite current concerns about infection with the new corona virus 2019 (COVID-19), prevention of its spread and its treatment, various lesions and conditions unrelated to this virus still occur. Prompt first aid can prevent an additional burden on the health system by taking care of simple ailments at the scene, rather than calling the ambulance system or transporting the victim to a hospital. This can also prevent unnecessary exposure to a possible infection with this virus.

There are several changes to current first aid protocols, most of which are related to preventing or minimizing the risk of viral transfer.

During the COVID-19 pandemic:

We consider that any victim has COVID-19 and we manage the situation as such. The victim may be asymptomatic but may nevertheless be a vector of disease transmission.

If the victim is a close relative of the rescuer (a member of the same household) and has already been infected with COVID-19, most likely the rescuer has already been exposed and may choose to provide first aid directly.

If the victim is not a close contact of the rescuer:

Follow nationally valid advice on social distancing and the use of personal protective equipment whenever possible.

The use of personal protective equipment (PPE - gloves, mask, eye protection, etc.) may not be possible for all first aid situations, but care must always be taken to protect both the victim and the rescuer.

Employees in key positions with responsibilities for care (or for providing first aid) must be properly equipped (with PPE) and must provide first aid without further delay.

If the victim is conscious and able to take care of himself following instructions, the rescuer must provide first aid from a safe distance $(2 \mathrm{~m})$. If the victim has a face protection / mask, he/she should be encouraged to wear it permanently.

If the victim is unconscious or unable to take care of themselves, then first aid may be needed directly. However, both the victim and the rescuer must be constantly aware of the risk of a viral transfer.

Sequence of actions when out-of-home care is required:

We immediately call for specialized medical help.

When possible, wear gloves when touching the victim. 
We wear a mask / face protection whenever possible, and we take care, as much as possible, of placing such protection on the victim's face.

We touch / manipulate only what is absolutely necessary, remembering that all surfaces around the victim can be virally contaminated.

We only offer essential first aid to limit exposure time. This may include controlling significant bleeding, applying a bandage, using an adrenaline auto-injector, checking consciousness by placing both hands on the victim's shoulders, shaking it lightly, and positioning the victim.

\subsection{Basic life support during the COVID-19 pandemic}

The rate of infection in Acute Severe Respiratory Distress Syndrome with the new Corona virus 2019 varies in Europe, and the general treatment recommendations of unconfirmed COVID-19 patients are adjusted according to the local risk assessment. For patients confirmed and suspected of COVID-19, the European Resuscitation Council recommends certain changes to the basic life support (BLS).

Rescuer's safety is important, regardless of whether he is a non-professional in the medical field or a medical professional [14]. In resuscitation, there is an inevitable trade-off between the risk to which the rescuer is exposed and the patient's benefit. Non-professional rescuers in the medical field usually assume a certain risk, as part of their profession, but try to keep it to a minimum. Risk assumption will depend on the relationship with the victim and one's own perception of risk. The key challenge in resuscitation during the COVID-19 pandemic is that both the precise risk to the rescuer and the actual benefit of the patient are unknown.

Some rescuers believe it is their duty to care for patients, making the most of their abilities and knowledge, regardless of risks. While doing all they can for one patient, rescuers need to be aware of their responsibility towards their relatives, colleagues and the rest of the community. Those who underestimate risk of transmitting the virus can become contagious to the rest of the medical team and the rest of society, adding to the pressure on the medical system.

Cardiopulmonary resuscitation presents a clear risk of transmitting contagious diseases, even if limited to chest compressions. Non-professional medical rescuers or those who arrive first on the spot, should protect themselves as best they can and avoid actions with a high risk of transmission, especially if they themselves are at increased risk of contracting the disease (elderly, suffering of chronic diseases, lung disease, heart disease).

Rescuers caring for the victim or living with the victim have probably already been exposed and may be more willing to perform resuscitation manoeuvres, regardless of potential increased risks.

In the current situation, analysis of each resuscitation attempt is extremely important, highlighting the evolution of the medical team, medical and ethical decisions and possible issues, such as personal protection and safety of rescuers.

Severe acute respiratory distress syndrome with corona virus 2 (SARS-CoV-2) has shifted the focus from patient safety to the safety of health care workers in the event of cardiorespiratory arrest. The increased risk of infection for the rescuer changes treatment approaches - including suspected and/or confirmed cases of COVID-19.

On the other hand, delays in initiating resuscitation can cost the lives of patients in cardiac arrest. High-quality resuscitation manoeuvres are still needed during a pandemic, being extremely important.

Taking into account the main mechanism of transmission for Sars-CoV-2 manifested by respiratory secretions, either directly from the patient or by touching contaminated surfaces, it is necessary to use personal protective equipment consisting of gloves, protective mask 
(type 3 Filter mask (FFP3) or N99 mask (FFP2 or N95), eye protection (full face shield / visor or goggles made of polycarbonate or equivalents).

"Cardio-respiratory arrest" is defined as the cessation of the heart's pump function and the cessation of respiration.

Cardiopulmonary resuscitation is a set of medical procedures that must be performed when, due to a cardiac / respiratory arrest, the oxygenation of the nerve cell is insufficient [14].

Resuscitation should only be performed on people in CARDIO RESPIRATORY ARREST (unconscious, not breathing).

It is extremely important for the brain to have a continuous flow of oxygen. Brain cells can only tolerate a short interruption of oxygen flow and after 3 minutes begin to appear lesions in the brain. After about 7 minutes these lesions may become irreversible.

In Basic Vital Support, cardio-pulmonary resuscitation aims to replace the pump function of the heart in cardiac arrest and maintain tissue oxygenation until the arrival of specialized medical crew, consisting of three components: Chest Compressions, Assisted Ventilation and Defibration.

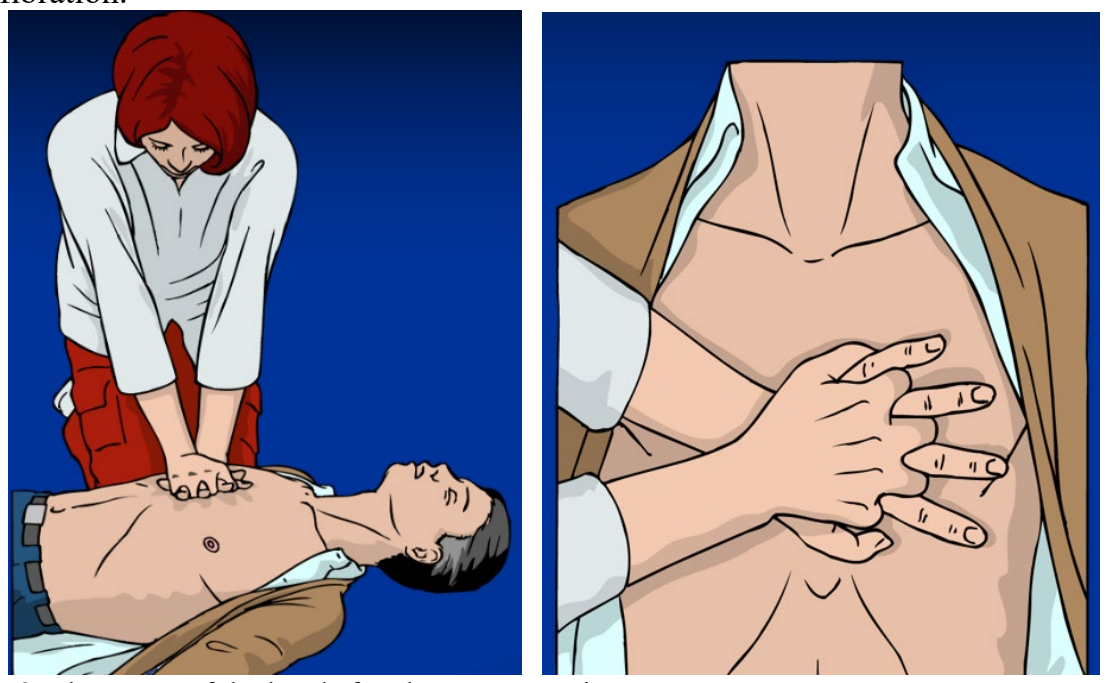

Fig. 2. Placement of the hands for chest compressions

Chest compressions cause reduced but critical blood flow to the brain and myocardium and increase the chances of successful defibrillation.

Position of hands and arms in performing chest compressions is especially important. Thus, ribs fractures / damage to internal organs are avoided and efficiency of the manoeuvre increases. The optimal technique for chest compressions involves: chest compressions with a frequency of $100-120$ / minute and a compression of at least $5 \mathrm{~cm}$ (in adults), but not more than $6 \mathrm{~cm}$, allowing the chest wall to return to normal after each compression; it is important to allocate the same time interval for compression and decompression.

It is necessary to continue resuscitation manoeuvres until the victim begins to breathe and there are signs of cardiac activity resumption, until the arrival of the medical team to provide emergency care or until the rescuer is too tired to continue.

Rescuers can be assisted in achieving compression rate and depth by feedback devices built into automatic external defibrillators or specially designed devices.

In case of assisted ventilation, if oxygen is available, it should be administered at a flow rate of 10 litres / minute. Airway permeability and lung ventilation are important components of cardio-respiratory resuscitation.

Defibrillation is the restoration of heart rhythm, disturbed by fibrillation phenomena and is defined as "the absence of ventricular fibrillation / ventricular tachycardia, without pulse 
5 seconds after applying the external electric shock", although the goal is to regain spontaneous circulation. That said, defibrillation is the passage of an electric current through the heart, in order to stop its chaotic or much too fast contraction, so that the natural stimulator takes control.

To achieve this, all defibrillators have three common characteristics: an energy source able of providing current, a capacitor that can be charged at a predetermined energy level, and two electrodes that are placed on the patient's chest.

The defibrillator electrodes can be placed in one of the following positions:

- one electrode to the right of the upper sternum, below the clavicle, above the nipple and the other electrode in the armpit (underarm) on the left mid-axillary line,

- one anterior electrode on the left hemithorax, above the heart and the other electrode on the back, behind the heart, inferior to the left shoulder blade (antero-posterior).

- one electrode placed in the axillary midline, and the other electrode on the back, over the right scapula (posterior-lateral).

- one electrode on the side walls of the chest (in the armpit), one on the right side and the other on the left side (bi-axillary).
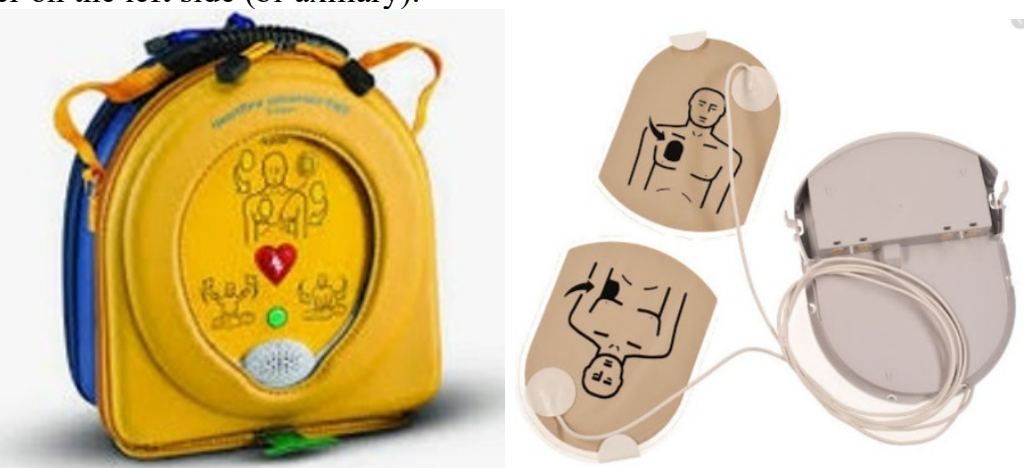

Fig. 3. Semiautomatic defibrillator

This manoeuvre is an important link in the chain of survival and also one of the two interventions proven to be truly effective in resuscitation along with chest compressions.

Cardio-pulmonary resuscitation manoeuvres will continue until the victim begins to breathe and there are signs of resumption of cardiac activity, until the arrival of the medical team to provide emergency care or until the rescuer shows signs of exhaustion (too tired to continue).

\section{Conclusions}

First aid is the initial care provided for an acute illness or trauma. The objectives of first aid include maintaining life, alleviating suffering, preventing further illness or injury and promoting recovery.

In case of an unconscious victim, it is necessary to immediately alert the emergency medical services by calling the unique number 112 . In case there's a single rescuer in the area, he will call the emergency medical service on his mobile phone (with the hands-free option) after which he will initiate cardio-respiratory resuscitation manoeuvres, assisted by the dispatcher.

Cardiopulmonary resuscitation manoeuvres will be delivered to anyone who does not respond to external stimuli or to victims with abnormal or absent breathing. Also, seizurelike movements may occur at the onset of cardiac arrest.

The victim will be evaluated after stopping the seizures, and, in case of lack of response and with absent or abnormal breathing, we will start cardio-pulmonary resuscitation (CPR) 
manoeuvres materialized by chest compressions with a frequency of $100-120 /$ minute followed by the administration of artificial breathing if we have the necessary equipment at hand.

The key challenge in cardio-respiratory resuscitation during the COVID-19 pandemic is to satisfactorily balance the risk of rescuers and benefits of victims. Rescuers will do everything they can for each victim, but at the same time, they must be aware of their responsibility towards their families, colleagues and the community.

\section{References}

1. https://www.osha.gov/sites/default/files/publications/OSHA3317first-aid.pdf

2. I. Urdă, Damages in industry. Emergency management (Agir, Bucharest, 2006)

3. http://www.onr.org.uk/intervention-records/1920/awe-aldermaston-19-201.htm

4. B. O. Alli, Fundamental principles of occupational health and safety (International Labour Office - Geneva: ILO, 2008)

5. https://www.osha.gov/sites/default/files/publications/OSHA3317first-aid.pdf

6. D. Pupăzan, et. all, Interventions and rescue in toxic / explosive / flammable environments (Insemex, Petroșani, 2020)

7. C. Ilie, G. A. Găman, D. Pupăzan, A. Irimia, A. Gireada, Intervention and rescue personnel assessment systems in toxic / explosive / flammable environments in the extractive industry (Focus, Petroșani, 2016)

8. D. Pupăzan, G. A. Găman, C. Ilie, A. Călămar, Intervention of rescue teams in toxic / explosive / flammable environments (Focus, Petroșani, 2016)

9. C. Nicolescu, I. Kovacs, C. Ilie, Training of intervention and rescue personnel in toxic / explosive / flammable environments (Insemex, Petroșani, 2018)

10. A. Gireadă, D. Pupăzan, C. Nicolescu, C. Ilie, A. Irimia, Optimizing the training process of intervention and rescue personnel in toxic and / explosive / flammable environments (Insemex, Petroşani, 2019)

11. A. Gireadă, D. Pupăzan, C. Nicolescu, C. Ilie, A. Irimia, Optimizing the training process of intervention and rescue personnel in toxic and / explosive / flammable environments (Insemex, Petroşani, 2019)

12. A. Irimia, G. A. Găman, D. Pupăzan. C. Ilie, C. Nicolescu, Environmental Engineering and Management Journal, 4, 831-837 (2019)

13. G. A. Gaman, D. Pupăzan, Procedures and regulations of intervention and operation formations in toxic / explosive / flammable environments (Insemex, Petroșani, 2009)

14. T. M. Olasveengen, F. Semeraro, et. al., Resuscitation, 161, 98-114 (2021) 